\title{
«PARIR POR LA BOCA». VICISITUDES DE UN TEMA SORPRENDENTE DEL IMAGINARIO DE LA COMADREJA
}

\author{
Isabel María de Barros Dias \\ Universidade Aberta de Lisboa
}

\section{RESUMEN}

Estudio de un motivo impactante recurrente en bestiarios: la comadreja que da a luz a través de la boca. Se muestran diferentes variantes de este topos y se debate su origen, probablemente una anécdota procedente de la mitología antigua, específicamente la historia de Galantis, sierva de Alcmena, que ayudó en el nacimiento de Hércules. Esta historia fue versificada por Ovidio de forma magistral y se encuentra también en Eliano. Su recuerdo persistió aún en algunos textos medievales.

PALABRAS CLAVE: comadreja; bestiario; motivos literarios; fuentes; mitología clásica

\begin{abstract}
Study of an impressive recurrent motif in Bestiaries: the weasel that gives birth through the mouth. Different variants of this topos are shown and its origin is discussed, probably a tale from Ancient mythology, specifically the story of Galanthis, servant of Alcmene, who helped the birth of Hercules. This story was masterfully versified by Ovid, and it can also be found in Aelianus. Its memory still lasted in some medieval texts.
\end{abstract}

KEY WORDS: weasel; bestiary; literary motifs; sources; classical mythology

Los bestiarios presentan a la comadreja como un animal singular en su manera de concebir y dar a luz. Algunas obras afirman que concibe por la oreja y pare por la boca, como el Bestiaire de Cambrai (siglo XIII). ${ }^{1}$ Otras dicen todo lo contrario, que es fecundada por la boca y da a luz por la oreja, como san Isidoro (siglo vII) en sus Etimologías, ${ }^{2}$ hecho que es calificado enseguida como una falsedad. Esta versión puede Recibido 7-X-2015. Aceptado: 22-XI-2015

1 «1. The nature of the weasel is that she conceives through the ear and gives birth through her mouth, and when her little ones have been killed, if she finds them, she looks for an herb which she brings them back to life.», «The Cambrai Bestiary» - traducción inglesa del texto en francés antiguo de E. B. Ham, editado en Modern Philology XXXVI/3, 1939, pp. 233-237 y reproducido en Guy R. Mermier (trad.), A Medieval Book of Beasts. Pierre de Beauvais' Bestiary, Lewiston/Queenston/Lampeter, The Edwin Mellen Press, 1992, pp. 311-315 (el pasaje citado está en la p. 311). Este texto se caracteriza por no presentar moralizaciones didácticas.

2 «3. Mustela dicta, quasi mus longus; nam telum a longitudine dictum. Haec ingenio subdola in domibus, ubi nutrit catulos suos, transfert mutatque sedem. Serpentes etiam et mures persequitur. Duo autem sunt genera mustelarum; alterum enim silvestre est distans magnitudine, quod Graeci ïxtıסas 
ser aún más compleja, cuando se hace depender el sexo de la cría de la oreja que la da a luz, como se verifica en el Physiologus latino. ${ }^{3}$ Otra posibilidad es la que presenta la oscilación entre la boca y la oreja sin elegir una de las variantes. Esto lo vemos en un testimonio del «bestiario de la segunda familia», ${ }^{4}$ fechado en $1180 .{ }^{5}$ Se trata de una rama textual procedente en gran medida de la obra isidoriana, razón por la que repite informaciones ya contenidas en las Etimologías, pero no califica como falso lo que se refiere al modo como concibe y da a luz ${ }^{6}$ el animal.

Según estudios desarrollados sobre las composiciones más antiguas, los Physiologus, en su origen redactados en griego, en fecha indefinida, en algún momento entre los siglos II y IV, la versión según la cual la comadreja concibe por la boca es posterior y ha conducido a cambios fundamentales en cuanto a la interpretación simbólica otorgada a la comadreja. ${ }^{8}$ La versión más antigua es la que presenta el

vocant; alterum in domibus oberrans. Falso autem opinantur qui dicunt mustelam ore concipere, aure effundere partum. // 3. Mustela (comadreja) es como si se dijera ratón largo, mus-telus, pues telus indica largura. Este animal muestra mucha astucia por lo que se refiere a las madrigueras en las que cuida de sus crías, pues muda y cambia de aposento. Persigue serpientes y ratones. Hay dos tipos de comadrejas: uno, que es silvestre y destaca por su mayor desarrollo, y al que los griegos llaman iktís; y otro, que vive en las casas. Es falsa la opinión de quienes afirman que la comadreja concibe por la boca y efectúa sus partos por la oreja.» Isidoro de Sevilla, Etimologias (ed. bilingüe de Jose Oroz Reta y Manuel A. Marcos Casquero), Madrid, BAC, 1983. El pasaje está en XII, 3, 3 (vol. II, pp. 78-e 79).

3 «The weasel has this nature: the female receives the seed of the male in her mouth and, having become pregnant, gives birth through her ears. If it happens, however, that she gives birth through the right ear, the young will be male, and if through the left ear, female.», Physiologus. A Medieval Book of Nature Lore (trad. Michael J. Curley), Chicago \& London, University of Chicago Press, 1979, p. 50.

4 Los bestiarios latinos se dividen generalmente en cuatro familias. Sobre las características específicas de los bestiarios de la segunda familia, véase la introducción de Willene B. Clark, A Medieval Book of Beasts. The Second-Family Bestiary: Commentary, Art, Text and Translation, Woodbridge, The Boydell Press, 2006.

5 Este manuscrito, editado por Willene B. Clark, está en London, en la British Library, MS. Add. 11283 (ed. Clark, 2006, p. 118).

6 «Duo autem earum genera sunt. Altera enim silvestre distans magnitudine; has Graeci ictidas vocant. Altera in domibus oberrans. Quidam dicunt eas aure concipere et ore generare; et contrario quidam dicunt eas ore concipere et aure generare. Dicuntur etiam peritae medicinae, ita ut si forte occisi fuerint eorum fetus, si invenire potuerint redivivos faciant. // Further, there are two types of weasels. One lives far away in the great forest; the Greeks call these ictidas. The other wanders about in homes. Some say that weasels conceive by the ear and give birth by the mouth, and conversely, some say that they conceive by mouth and give birth through the ear. They are said to be skilled in medicine, so that if perchance their young should be killed, they can revive them if they can find them.» Clark, 2006 pp. 161-162.

7 Sobre las distintas hipótesis de datación, sus argumentos y fragilidades, véase la introducción de Michael J. Curley a Physiologus... (pp. XVII-Xx), así como la introducción de Arnaud Zucker al physiologus griego [Physiologos. Le bestiaire des bestiaires (trad. y ed. Arnaud Zucker), Grenoble, Jérôme Millon, 2005, pp. 12-13].

${ }^{8}$ Sobre el carácter ejemplar, moral y alegórico de los animales en los bestiarios véase, por ejemplo, el libro colectivo de Jacques Berlioz y Marie Anne Polo de Beaulieu (dir.), L'animal exemplaire au Moyen Âge (Ve-XVe siècles), Rennes, Presses Universitaires de Rennes, 1999. Para ilustrar la flexibilidad simbólica y la consecuente inestabilidad y reversibilidad de las interpretaciones alegóricas del bestiario, véase el artículo de Arnaud Zucker, «Morale du Physiologos: le symbolisme animal dans le christianisme an- 
parto por la boca, perteneciente a la tradición griega, y que simboliza el nacimiento de la palabra; mientras que la tradición inversa es más reciente y tiene un significado negativo asociado a la esterilidad espiritual. ${ }^{9}$

Esta interpretación negativa es evidente en el «bestiario de la segunda familia» mencionado anteriormente pues, mientras presenta las dos versiones relativas a los lugares de concepción y parto de la comadreja, se fija asimismo en la lectura simbólica según la cual este animal representa a los hipócritas que escuchan la palabra de Dios, pero que, distraídos por el mundo, se olvidan de lo que han oído:

Significant autem aliquantos qui libenter quidem audiunt Divini Verbi semen, sed amore terrenarum rerum detenti praeterimittunt et dissimulant quod audierint.

Now, they symbolize the many people who gladly hear a kernel of the Divine Word, but distracted by the love of earthly things, they overlook and neglect what they heard. [Clark, (ed. / trad.) 2006, p. 118]

La lectura negativa parece ser la más común, divulgada en múltiples bestiarios que coinciden en asimilar la concepción y parto de la comadreja a las personas que no siguen la palabra de Dios, la olvidan o desobedecen. Esta noción se ve claramente en textos como el Bestiaire de Gervaise, una obra en verso de finales del siglo XII - inicios del XIII; ${ }^{10}$ el Bestiaire divin de Guillaume, Clérigo de Normandía, obra también en verso y

cien (IIe-Ve s.)», Rursus, 2, 2007. Disponible en http://rursus.revues.org/142 DOI: 10.4000/rursus.142 [consultado 01-05-2015].

9 Véase lo que dice Zucker en sus comentarios a la entrada sobre la comadreja en los physiologus griegos: «Sous sa forme originelle ou «correcte» le circuit génératif de la belette peut apparaître parfaitement positif car il mime symboliquement le travail intérieur de la parole capable de transformer le disciple en apôtre, le catéchumène en prédicateur. [...] Or justement c'est le propre du Physiologos, dans lequel la belette est symbole d'inconsistance frivole et de stérilité spirituelle, d'inverser l'ordre de la parole féconde, en allant au rebours de la tradition grecque unanime. Et c'est cette inversion qui rend la belette négative et fait de son enfantement... un avortement de la parole divine» (Physiologos..., p. 152). Este punto de vista lo comparte Alexandre Vermeille, Physiologus. De l'Orient à l'Occident. Un patchwork multiculturel au service de l'Écriture, Mémoire de latin-Université de Neuchâtel, 2006, p. 69. El hecho de que la versión según la cual la comadreja empreña por las orejas sea la más antigua puede conjeturarse también a partir de la versión latina del physiologus que, mientras siguiendo la formulación más reciente (véase la cita en la nota 3) aún afirma: «Wicked things are engendered through the ears», (Physiologus..., 1979, p. 50), lo que puede constituir una huella de una versión anterior contraria. Zucker sugiere la posibilidad de que una confusión lingüística esté en el origen de esta inversión: «Si on la considère comme un «malentendu» on peut l'expliquer par une confusion linguistique ou une contamination: ore (par la bouche) a pu devenir aure (par l'oreille) d'autant que l'oreille en grec ous est très proche de la bouche latine os; d'autre part, la conception orale de la vipère, voire celle que l'on prêtait aux poissons gobeurs de laitance, a pu rejaillir sur la belette et provoquer cette inversion.» Physiologos..., p. 151 - pero esta explicación choca con el hecho de que el physiologus griego, que el propio Zucker traduce, ya presenta la versión de la concepción por la boca y parto por las orejas... (cf. Physiologos..., p. 150).

10 «Petite beste est la belete; / La loi dit qu'ele n'est pas nete, / Por ce n'en doit un pas mengier, / 1140 Que ançois qu'ele doie emprenier / Et ses maules a li atoche, / Sen germen reçoit en sa boche; / Et encor fait grenor mervoille, / 1144 Que ele feone per l'oroille. / Per la beste entendre poons / Ces qui vunt es religions / Voluntiers et qui le sermon / 1148 Reçoivent a devocion, / Puis metent tot en obliance, / Ne sunt mie fer(t) en creance.» — «Le Bestiaire de Gervaise» (ed. Paul Meyer), Romania, 1, 1872, pp. 420-443 (el texto citado está en la p. 441). 
de inicios del siglo XIII; ${ }^{11}$ o aún el Bestiario de Pierre de Beauvais, en su versión breve, datada de inicios del siglo XIII:

Phisiologues dit quelle rechoit la semenche del masle par la bouche, ensi la dedens soy. El tens quelle doit foener, elle le rent par loreille. Ansi font les fiaubles en dieu qui volentiers rechoivent la semenche $\mathrm{d}(\mathrm{e})$ la parolle dieu, mais li deviennent puis inobedient, il entrelaissent ce quil ont oy de dieu.

Physiologus says that the female receives the seed of the male in her mouth and she swallows it. Then when she is about to give birth, she gives birth through her ear. Similar to the weasel are the faithful who receive most willingly the word of God, but who later abandon their faith and disregard what God has told them. ${ }^{12}$

Hay, sin embargo, bestiarios que interpretan de modo diverso las características de la comadreja que investigamos. Esto sucede con el Bestiaire d'Amour de Richard de Fournival, de mediados del siglo XIII, que recupera la versión más antigua de la concepción por la oreja y del dar a la luz por la boca para referirse, por un lado, al gusto de escuchar y reproducir palabras bonitas ${ }^{13} \mathrm{y}$, por el otro lado, en la «Réponse de la Dame», para referirse a los calumniadores y a los rumores que pueden llevar el reino a la ruina. ${ }^{14}$

11 «XXVIII. De la belete // De la belette est grand mervelle; / Quer el effante par l'orelle, / Et par mi la boche receit / La semence par quei conceit. / De malle, quant ele l'aproche, / Prent la semence par la boche, / 2250 Que dedenz son ventre norrist, / Et par mi l'orelle s'en ist. / Ceste petite beste mue, / Por ce ses founceaus remue / Sovente feiz de leu en leu, / Ne tient pas une place en feu. / Les serpenz moult durement het, / De la les chace ou el les set. / / A cest sunt aconparagie / Plusors qui sunt encoragie / De bien oir, de Deu servir; / De la parole Deu oir / Sunt curious, a le entendent, / En lor corage a Deu se rendent / Et commencent bien a ovrer, / A Deu servir et a amer; / Et en petit d'ore recreient, / Et ce que il aiment, mescreient, / Et ne sunt mie obedienz / A fere ses comandemenz, / Si cum il li orent pramis.» - Le Bestiaire divin de Guillaume clerc de Normandie, trouvère du XIIIe siècle (ed. C. Hippeau), Ginebra, Slatkine, 1970, pp. 267-268.

12 Transcripción del manuscrito COD. 32 del Groot Seminarie Mechelen, Bélgica (testimonio - fechado del siglo xv- de la versión breve del Bestiario de Pierre de Beauvais). Este texto y su traducción están en Guy R. Mermier (trad.), A Medieval Book of Beasts. Pierre de Beauvais' Bestiary, Lewiston / Queenston / Lampeter, The Edwin Mellen Press, 1992, p. 285 (versión en francés antiguo) y 153-154 (traducción en inglés).

13 «La Mostoile. // Aussi com la Mostoile, qui par l'orelle conçoit et par la bouche enfante. Et teil nature font tieus femes; jà soit q'eles aient oïs tant beaus mos qe lor samble que les doivent amer, et qu'eles ont aussi coume conciut par l'orelle, si s'en délivrent par la bouche à un escondit, et salent volentiers en autres paroles par costume, aussi coume elles doutassent d'estre prisses. Tout en tel maniere come la mostoile meesmes qui porte ses faons, quant elle les a enfantés, par paor de perdre; et cette daerraine nature de la mostoile si est une desesperance d'amors, c'on ne voelle oïr parler de çou que grignor mestier puet avoir et toudis voelle-on parler d'el.» Richard de Fournival, Le Bestiaire d'amour suivi de la réponse de la Dame (ed. C. Hippeau), Ginebra, Slatkine, 1978, p. 14.

14 «La Mostoile. // Dont ore me sovient à cesti chose que jou ai entendut que la Mostoile si conçoit par l'oreille et faone par la bouche. Certes de ceste nature de Mostoile voel-jou bien qu'il me soviegne. Car concoivres par l'orelle et faoner par la bouche est granz senefiance. Car jou di que conçoivres si est une chose qui mout fait que on ne conçoive chose dont on n'ait au phaoner à sousfrir. / / Ha Dex! come li auquant i deveroient bien prendre garde! Car tel gent $\mathrm{i}$ a qui conçoivent par eus meismes aucunes choses que il oent, dont li enfanters est si griés et si perilleus que merveille. Car li aucun getent teil parole hors, dont miols venroit que il le portassent tant que il en crevassent à fines certes. / / Car veritez est que pis ne puet li hom faire ne la feme que de son phaoner, c'est de dire chose qui n'est covignable et dont nus roiaumes puet estre destruis. Dex! si en ai teil paor, que je ne m'en sai mie bien conseillier. Car je di que 
Por fin un ejemplo peninsular, el Bestiaris catalán, que también toma la versión más antigua y ofrece un significado positivo, pues se refiere a los buenos predicadores que siembran la palabra de Dios a los oídos de los hombres, aunque pocos correspondan:

De la natura de la mostela e de la sua significació

La mostela si és una bestiola petita [qui és molt asperta], e ha en si una fort natura: que ella concep per la orella e infanta per la bocha; e fa sos fills morts, e diu hom que ella coneix una erba la qual los fa tornar vius.

Aquesta mustela significa los bons preÿcadors qui preÿquen la paraula dels Evangelis, e de les pístoles, de les profecies, en les orelles dels hòmens del món; car ells preÿquen caritat, so és, amar Déu sobra totes coses del món, e son proïsme axí com si mateix; e preÿquen pobresa, e pau, e humilitat, e castedat e dretura. E són quaix pochs hòmens qui vulen usar ni perseverar en aquestes virtuts, ans si ho han entès en lo preÿch, mantinent los és oblidat, car lo cor han mortificat. Emperó, bé s'esdevé que a vegades va remembrant a alguns hòmens de les bones paraules que hauran hoÿdes, les quals los eran oblidades; e van per la erba qui fa ressucitar bones paraules de Déu, la qual erba és la remembransa de la glòria de peradís e la remembransa de les penes de infern, car sapiats per sert que aquestes dues coses són aquelles qui més fan remembrar los peccadors en fer bé..$^{15}$

El carácter poco común e incluso sorprendente de este motivo, al que se suma la inestabilidad de su transmisión y las consecuentes fluctuaciones interpretativas, nos ha estimulado a buscar explicaciones que precedan a las interpretaciones literarias y morales ya conocidas. Una búsqueda a través de los comentarios existentes trajo pocas aclaraciones sobre el origen de este fenómeno. Willene B. Clark, en nota, refiriéndose a otra nota de Jacques André, editor de la obra de Isidoro (edición de las Belles Lettres, de 1986) sugiere que en el origen de este prodigio esté la equivocada observación de la vida animal, más precisamente, el hecho de que el macho muerda la piel del cuello de la hembra durante el apareamiento. ${ }^{16}$ Esta explicación nos parece poco creíble dada la proximidad con el mundo animal que se verificaba en épocas más lejanas, lo que no es compatible con un error tan grosero de observación, al que se suma que la costumbre de morder la piel del cuello de la hembra es común en distintas especies animales a las que no han sido asignadas ninguna particularidad de concepción ni de parto.

se il est chose que jou die aucune parole que jou aie conciut par l'oreille, jou me doute molt que ele ne portie venim et que morir ne la coviégne. / A Aussi que on compte des faons à la Mostoile, que quant on li ocist ses mostelos et on li remet arrière en son liu, que la mère seit de sa nature que ele les resuscite.» Richard de Fournival, Le Bestiaire d'amour suivi de la réponse de la Dame (ed. de C. Hippeau), Ginebra, Slatkine, 1978, pp. 66-67.

15 Bestiaris (a cura de Saverio Panunzio), Barcelona, Barcino, 1963 (vol. I), pp. 76-77 - edición de la versión del testimonio A (ms. 75 de la Biblioteca Universitària de Barcelona, con letra del siglo XV).

16 «Isidore says such notions are false. André, ed. Isidorus, 126n 199, surveys the history of this lore. The mating through the ear may be a misapprehension of the process by which the male takes the female's neck fur in his mouth and drags her about before mating; see Grzimek, Encyclopedia III, 394». (Clark, 2006, p. 162n). 
Una explicación semejante fue producida para explicar el parto por la boca, apoyándose en el argumento de que las crías de la comadreja son muy pequeñitas y que su madre las carga en la boca, lo que podría dar origen a una confusión con un parto. ${ }^{17}$ Pero también este es un hábito común en muchos animales... A estos apuntes se suma el hecho de que no encontramos estudios específicos sobre la presencia de la comadreja en los bestiarios, solo comentarios dispersos y en su mayor parte breves y no muy desarrollados..$^{18}$ Por eso, parece haber espacio para la presentación de otras hipótesis que busquen aclarar un poco mejor el origen de estas características tan extravagantes.

Podemos todavía pensar que estos pasajes aluden de modo subliminal a prácticas sexuales no convencionales. Esta hipótesis es compatible con el carácter sucio que a menudo se atribuye a la comadreja, además de que los textos subrayan el hecho de que este animal recibe el semen en la boca. Sin embargo, las interpretaciones moralizantes que se proporcionan no desarrollan esta posibilidad, excepto, quizás, el bestiario de Philippe de Thaün, de inicios del siglo XIII, que utiliza la expresión contra natura, pero refiriéndose, en términos generales, a contradicciones, como escuchar por la boca y hablar por la oreja:

Il est une bestete

$\mathrm{Ki}$ at num mustelete,

Dunt nostre lei defent

Qu'om n'en manjuce nient.

E Phisiologus

De la mustele dit plus:

La semence que dune

Sis masles, dunt feüne,

En sa buche receit,

En tel guise cunceit;

E quant feünerat

Par l'oreille naistrat.

Grant chose signefie,

\footnotetext{
17 Véase el siguiente comentario de Zucker: «On ne peut reconstituer sûrement l'étiologie de cette croyance, mais Aristote ([Histoire des animaux] 756b), qui rejetait déjà cette fable attribuée à Anaxagore a peut-être raison d'y voir le fruit d'une observation hâtive: «comme la belette fait des petits minuscules, dit-il, [...] et comme elle les transporte souvent dans sa gueule c'est de là qu' est venue cette légende». A ce mirage s'est ajoutée, dans un mélange au mystérieux dosage qui fait la saveur des personnages du bestiaire, une confusion avec son homonyme marin, la «belette de mer», un squale (galeos) qui passait pour reprendre dans sa bouche ses petits lorsqu'il croisait un danger, puis les recracher, «comme s'il les mettait au monde à nouveau» (Élien, I, 17)» Physiologos..., p. 151-152.

18 El ejemplo más notable de esta ausencia es el hecho de que la comadreja no aparece en el diccionario de Gaston Duchet Suchaux y Michel Pastoureau, Le Bestiaire médiéval. Dictionnaire historique et bibliographique, Paris, Le Léopard d'or, 2002.
} 
Oëz l'allegorie.

Issi funt mainte gent

Volenterivement,

Oent le sermun Dé

Qu'il unt puis en vilté;

Cil funt diversement

E eschars sunt de gent:

Ki par buche l'entent

Par l'oreille le rent,

Icil ki par buche ot

Par l'oreille dit mot;

Ço est del serf pulent

Ki cuntre Dé se prent,

Ki fait cuntre nature,

Si cum dit escripture. ${ }^{19}$

Además, la mayoría de estos bestiarios fueron redactados por clérigos y las prácticas menos convencionales no solo fueron criticadas con severidad y castigadas por la Iglesia, sino también silenciadas. Esta actitud es muy clara en los penitenciales, que pueden referir en detalle las variantes del pecado de la lujuria a fin de especificar la respectiva penitencia, pero frecuentemente también advierten que el interrogatorio del pecador no debe ser demasiado explícito para no despertar la curiosidad..$^{20}$

Bastante más probable es otra línea de informaciones que encontramos en los comentarios de los physiologus griegos y latinos. Zucker (Physiologos..., p. 151) recuerda la historia de Galante, la sierva de Alcmena que ayudó al nacimiento de Hércules. Según este relato, Alcmena no puede dar a luz porque la diosa de las embarazadas, en connivencia con Juno, no lo permite. La diosa se sentó delante de la puerta con las piernas entrelazadas, los dedos entrelazados, y pronunció palabras de hechizo que retuvieron el parto. Galante se da cuenta de que había algo extraño y desafía a la diosa diciendo que su señora ya había dado a la luz. La diosa de las embarazadas se perturbó y desentrelazó las piernas y las manos, y en ese momento Alcmena pudo dar a luz. Galante se da cuenta de que había engañado a la diosa y se ríe. Enseguida es castigada

19 Le Bestiaire de Philippe de Thaün (ed de Emmanuel Walberg), Lund, H.J. Möller - H. Welter, 1900, pp. 45-46 (vv. 1217-1244).

20 La necesidad de preguntar cuidadosamente aparece en el Livro das Confissões de Martín Pérez, de 1316 (ed. José Barbosa Machado y Fernando Torres Moreira, Matosinhos, Pena Perfeita, 2005 - p. 67). Ya el Tratado de Confissom, impreso en Chaves, en 1489, refiere en varias ocasiones (vol. I, pp. 38, 39-40, 47-51, 62-64, 87-91) el sexto mandamiento, y detalla sus posibilidades, determinando las penitencias que deben aplicarse a cada caso - Tratado de Confissom (ed. José Barbosa Machado), Braga, APPACDM, 2003 , vol. I. 
y metamorfoseada en un animal del cual se dice que es diligente, rubio, que asiste a los hogares y, por haber ayudado al parto con boca mentirosa, da a luz por la boca. ${ }^{21}$

Es por supuesto imposible establecer una filiación directa entre los bestiarios y Ovidio. Curley, que también refiere el pasaje de Ovidio, junto con otros autores, sobre todo posteriores (Physiologus..., p. 86), habla de «análogos clásicos» (p. 68) pues, de hecho, también es imposible no darse cuenta de las similitudes.

Entre los autores más antiguos, Aristóteles parece no referirse a las características de la concepción y parto de la comadreja. ${ }^{22}$ Lo mismo sucede con Plinio el Viejo (23-79 d.C.), que es muy poco posterior a Ovidio (43 a.C.-18 d.C.) ${ }^{23}$ y con Solino (que vivió en el siglo III o IV). ${ }^{24}$ Otros ya mencionan las particularidades de la comadreja que nos

21 «una ministrarum, media de plebe, Galanthis, / flaua comas, aderat, faciendis strenua iussis, / officiis dilecta suis. ea sensit iniqua / nescio quid Iunone geri, dumque exit et intrat / saepe fores, diuam residentem uidit in ara / bracchiaque in genibus digitis conexa tenentem, / et «quaecumque es,» ait «dominae gratare. leuata est / Argolis Alcmene, potiturque puerpera uoto.» / exsiluit, iunctasque manus pauefacta remisit / diua potens uteri: uinclis leuor ipsa remissis. / numine decepto risisse Galanthida fama est. / ridentem prensamque ipsis dea saeua capillis / traxit, et e terra corpus releuare uolentem / arcuit, inque pedes mutauit bracchia primos. / strenuitas antiqua manet; nec terga colorem / amisere suum: forma est diuersa priori. / quae quia mendaci parientem iuuerat ore, / ore parit nostrasque domus, ut et ante, frequentat.»» - Ovidio, Metamorfoses (ed. bilingüe de Domingos Lucas Dias), Lisboa, Nova Vega, 2008, vol. II, p. 80 (libro IX).

22 En la Historia de los Animales, Aristóteles (s. IV a.C.) se limita a comentar con brevedad que la comadreja es un animal con órganos sexuales óseos (libro II) y la nombra también cuando habla de las antipatías entre animales y las maneras como pelean (libro IX) - esta búsqueda solo ha considerado los libros I a IX y no el libro X, apócrifo. Histoire des Animaux d'Aristote (trad. fr. par J. Barthélemy-Saint Hilaire), Paris, Hachette, 1883 -disponible en: http:/ / remacle.org/bloodwolf/philosophes/Aristote/ tableanimaux.htm [consultado 13-06-2015].

23 En la Historia Natural, Plinio refiere la comadreja en diversas ocasiones: cuando presenta su capacidad para matar al basilisco con su (mal) olor (VIII, 33), cuando refiere las antipatías entre animales $(X, 95)$ y sus características fisiológicas (XI,70 y XI, 109). La comadreja es referida sobre todo cuando Plinio habla de medicinas inspiradas en el comportamiento de los animales y de recetas y procedimientos que incluyen animales o partes de los mismos (VIII, 41; XVIII, 45; XXVIII, 27; XXIX, 16, 27, 32, 33, 36 e 38; XXX, 12, 13, 16, 23, 27, 29, 36, 39, 43 e 50). Pliny the Elder, The Natural History (ed. John Bostock y H.T.Riley), London, Taylor and Francis, Red Lion Court, Fleet Street, 1855 - disponible en «Perseus Digital Library» (Gregory R. Crane, editor-in-chief, Tufts University) Disponible en: http://data.perseus.org/citations/urn:cts:latinLit:phi0978.phi001.perseus-eng1:1. dedication [consultado 01-05-2015]. Esta vertiente más práctica se encuentra todavía en Vicente de Beauvais, Speculum Naturale, libro XIX, cuyos cap. cXXXIII y cXXXIIII son dedicados a «De mustela» (fl. 245r-v). Cotejando otros diversos autores, Vicente de Beauvais menciona tres veces que la comadreja concibe por la boca y da a luz por la oreja, pero no pone de manifiesto el hecho, ni hace lecturas interpretativas, se limita a comentar (probablemente en secuencia de san Isidoro) que son frívolos quienes dicen eso (fl. 245v, col. a). De hecho, como Plinio (que es referido de manera explícita), atribuye más importancia al conocimiento y al uso médico de la comadreja, tema al cual dedica un tercer capítulo (cap. cxxxv — «De medicinis mustelis»-fl. 245v-col.a), además de lo que ya había dicho sobre la materia en los dos capítulos anteriores. Vincentius Bellovacensis, Speculum naturale,Venetiis, Hermannus Liechtenstein, 1494.

24 En la Collectanea rerum memorabilium, este autor solo refiere el hecho de que la comadreja derrota al basilisco. C. Ivlii Solini, Collectanea Rervm Memorabilivm (ed. Th. Mommsen), Berolinum, Weidmann, 1895 (p. 127). 
ocupan, como Plutarco (46-120 d.C.) $)^{25}$ y sobre todo Claudio Eliano (c. 175- c. 235). Curiosamente Eliano no es referido por Zucker ni por Curley por lo que respecta a la comadreja, a pesar de que este autor es uno de los que más informaciones comparte con los physiologus y bestiarios ${ }^{26}$. En su libro Historia de los animales, escrito originalmente en griego, y contemporáneo de las fechas más remotas que se atribuyen a la composición de los primeros physiologus, se compilan pequeñas anécdotas sobre animales de fuente sobre todo libresca, además de conocimientos tradicionales. La comadreja es mentada en varias ocasiones, ${ }^{27}$ pero aún no se habla de ninguna particularidad de concepción o de parto. A pesar de eso, hay dos pasajes que consideramos significativos y que deben de ser valorados:

Pero los tebanos, que son griegos, veneran, según tengo oído, a la comadreja y hasta llegan a decir que este animal fue nodriza de Heracles o, si su condición de nodriza debe quedar totalmente descartada, que, por lo menos, cuando Alcmena estaba acostada con dolores de parto y no podía dar a luz, la comadreja pasó por delante de ella y, con sólo eso, solucionó los impedimentos del parto, con lo que Heracles salió y hasta empezó a andar a gatas. (Libro XII, 5, p. 466)

He oído decir que la comadreja de tierra fue una mujer que se llamaba así y que era hechicera y bruja. También ha llegado a mis oídos que la entonces mujer de nombre Comadreja era tremendamente desenfrenada y que padecía un apetito sexual desordenado. Tampoco me pasó inadvertido esto, que la cólera de la diosa Hécate la trocó en este animal maligno. (Libro XV, 11, p. 571)

Una vez más, es imposible hablar de fuentes o de influencias directas. Pero tenemos aquí una serie de puntos que conectan características atribuidas a la comadreja en los physiologus y bestiarios con la historia del nacimiento de Hércules, e incluso con ideas menos explícitas pero quizás significativas, como el reproche sexual o el detalle de la similitud entre el nombre de la mujer y el nombre del animal. ${ }^{28}$

25 «There are still many people who believe and declare that the weasel conceives through its ear and brings forth its young by way of the mouth, and that this is a parallel of the generation of speech.» Plutarch, On Isis and Osiris, 381 -trad. de Plutarco por Frank Cole Babbitt - , ed. Loeb (1936) disponible en: http:/ / penelope.uchicago.edu/Thayer/E/Roman/Texts/Plutarch/Moralia/Isis_and_Osiris*/ home.html [consultado 13-06-2015].

26 Eliano es uno de los autores más referidos por Curley en las listas de «análogos clásicos», mientras que de Ovidio se hace mucha menos mención - Physiologus..., pp. 68-92. Zucker (Physiologos... p. 26) se refiere a las tradiciones que coinciden en el physiologo y menciona a Eliano (corriente de la zoología griega), a pesar de subrayar que se trata de paralelismos y que es imposible hablar de filiaciones. Este autor tampoco refiere a Ovidio en este apartado.

27 La comadreja aparece en los libros IV, 14; VII, 8; IX, 41; XI, 19; XII, 5; XV, 11 y 26. Se hacen breves referencias a que pelea con el olor, los sonidos que hace, el hecho de que, como los ratones, presagian tormentas y deslizamientos de tierras... Véase Claudio Eliano, Historia de los animales (trad. de José Vara Donado), Madrid, Akal, 1989.

28 Agradezco a Bernard Darbord el haber llamado la atención hacia el hecho de que el término «comadreja» deriva de «comadre», que además de madrina, todavía designa una mujer que asiste a las parturientas. El nombre en portugués, «doninha», como señaló Ana Paiva Morais, puede leerse como «pequeña dueña», lo que puede apuntar en la misma dirección. Además véase el siguiente comentario de Zucker: «la nourrice d'Alcmène, (appelée Gal-inthias ou Galè = "belette”)» Physiologos..., p. 151. Así, a pesar de que en estas lenguas románicas la comadreja tenga nombres tan diversos («doninha», "coma- 
De acuerdo con el contexto intelectual medieval, las fuentes escritas eran muy valoradas, especialmente si el autor era una autoridad. A pesar de eso, no podemos encontrar una filiación específica entre physiologus o bestiarios y los autores cotejados, lo que nos hace pensar que la historia de Galante sería del conocimiento general y que, cuando los primeros physiologus fueron redactados, se habría verificado una inspiración en ese tema, mediante remodelación y adaptación a sus intentos de moralización cristiana. $^{29}$

Es sabido que Ovidio dio una forma poética magistral a historias que corrían en su tiempo. Por otro lado, los pasajes de Eliano citados, dos siglos posteriores a Ovidio y posibles contemporáneos de los primeros physiologus griegos, pueden ser entendidos ya como testimonios de tradiciones dispersas y un poco brumosas (véase la recurrencia a la expresión formular del «oír decir» en los dos pasajes). Además, esta idea de que se tratara de una historia conectada con la mitología antigua que se popularizó en la tradición oral es compatible con la presencia del tema de la concepción maravillosa en la literatura tradicional, lo que no sería caso inédito (véase el ejemplo de la historia de Eros y Psique, tan recurrente en los cuentos tradicionales).

Por otra parte, es verdad también que la historia de la sierva de Alcmena solo explica la mitad del díptico. La idea de la concepción por la oreja podría haberse agregado al tema del dar a luz por la boca en un proceso acumulativo de motivos, bastante común en todas las épocas, y sobre todo en las historias tradicionales. ${ }^{30}$ De

dreja», «belette»), parece existir coincidencia al nivel del imaginario subyacente.

29 Véase lo que dice Curley: «The general view was that the author played a passive role in collecting the preexistent legends and an active one in composing their interpretations.» (Physiologus... p.xxII).

30 Véase el motivo popular de la concepción maravillosa - agradezco las indicaciones dadas a este respecto por María Jesús Lacarra: su presencia en el catálogo de Aarne y Thompson (Antti Aarne y Stith Thompson, The Types of the Folktale. A Classification and Bibliography, Helsinki, Academia Scientiarum Fennica, 1987 - véase motivos como el 705-Conception from eating fish; 707A-Virgin to Bear Child; 708The Wonder-Child o el 1362-The Snow-Child) y el importante artículo de Daniel Devoto sobre varios tipos (clásicos, tradicionales y de la literatura de autor) de concepciones sobrenaturales (Daniel Devoto, «Pisó yerba enconada», Textos y Contextos. Estudios sobre la tradición, Madrid, Gredos, 1974, pp. 11-46). Este motivo de la concepción, presente en la literatura tradicional, también tiene correspondientes cristianizados, siendo el ejemplo mejor conocido la concepción de Jesús en María por el verbo divino en la Anunciación - agradezco la sugerencia de este ejemplo a César García de Lucas, así como compartir su artículo «Iconotropía y literatura medieval», Carlos Alvar (coord.), Estudios de Literatura medieval en la Península Ibérica, San Millán de la Cogolla, Cilengua, 2015, pp. 593-605, donde el autor refiere un curioso pasaje del Libro del Santo Grial en el que, junto con algunas imágenes iconográficas, se refiere de manera explícita que el Espíritu Santo bajó al vientre de la Virgen María a través de la oreja (p. 603). Sobre la concepción auricular, véase también lo que dice Zucker: «Cette voie fut de fait empruntée, timidement et marginalement, par des textes apocryphes comme le Livre de Jean l'Evangéliste ou le texte arménien de l'Évangile de l>enfance. Ils mirent la belette en rapport avec l'enfantement virginal de Marie, à laquelle ils attribuèrent une conception auriculaire qui eut quelque écho au Moyen-Âge.» (Physiologos... p. 153). Por otro lado, el fenómeno estudiado en el artículo de García de Lucas es importante para explicar el proceso de cristianización de imágenes (en sentido lato, iconográficas y textuales) de la tradición Antigua existentes en el bestiario, lo que sería el caso de las características de la comadreja aquí estudiadas, pues, 
modo similar a lo que sucede con los textos tradicionales, también los physiologus y los bestiarios son textos de construcción acumulativa con una tradición textual muy larga. ${ }^{31}$

Además, la historia de Galante no fue olvidada en el período medieval, como prueba un pasaje de la General estoria de Afonso X, del siglo XIII, coetáneo de un momento de gran expansión de bestiarios, donde se identifica de modo explícito la transformación de Galante en comadreja:

En todo esto avié ý una sirvienta muy privada de Almena, e era esta manceba muncho entendida, e avía nombre Galante. E en yendo a aquel templo munchas vezes vio a aquella muger seer allí toda vía con sus manos de aquella guisa e que se non movié de allí, e mesuró que Juno podrié seer aquella que seyé allí desemejada faziendo aquellos fechizos contra Almena, e vino del templo e entró al palacio, e falló a Almena su señora en muy grand pena como antes, e non dixo nada en casa, e salió luego a la puerta e començó a dar grandes bozes e a fazer semejança de grand alegría, e a dezir que librada era su señora Almena, grado a los sus dioses. E Juno cuando lo oyó cuidó que en el su encantamiento e en aquel su saber que non oviera fuerça ninguna nin poder de aquella vez, si non que non sería aquello, e pesóle muy de coraçón e tóvose por maltrecha en ello, e soltó luego las manos unas de otras e decendió el inojo que tenié sobre el otro, e desfízose el fechizo, e encaeció luego Almena de dos fijos varones. E aquella manceba Galante pues que esto ovo fecho en la calle entró al palacio e falló a su señora encaecida, e tornóse luego a la puerta de cabo e començó a contar a los que venién esta alegría, e a los que pasavan por la calle, cómo por aquella palabra que ella dixiera que se desfizieran los fechizos que avían fechos a su señora que estava de parto e en ora de muerte, e que encaeciera luego e era librada e parida de dos fijos varones. E Juno cuando oyó esta artería que fiziera Galant tóvose por enartada d'ella e non del su saber, e dizen en este lugar los gentiles en sus razones que encantó Juno a Galante por este engaño que le fizo, e porque dixera ella por la boca que parida era ya su señora, non aviendo aún parido, e le fiziera desfazer su encantamento con que se quería vengar de Almena, que la tornó en mostelilla, mas esto que los abtores dixeron non fue ál si non que llamó Juno a Galante mostelilla por estas razones: la una por aquello que dixo que avié parido Almena, e lo ál porque entrava e salié e andava apresurada a todas partes con cuita de su señora, e como la mostolilla á natura de andar así muy privado a aquella manera, ca fallamos que después fue Galante partera muy preciada en aquella tierra toda por este fecho, e la llamavan las grandes dueñas para esto e la amavan muncho e le fazién muncho de algo por ello, ca dezién que avenié ý muncho bien. ${ }^{32}$

como dice el autor: «un proceso iconotrópico típico: la representación de un ser ajeno al cristianismo acabó por explicarse de acuerdo con la nueva ortodoxia religiosa.» (García de Lucas, «Iconotropía...», p. 601).

31 Cuando Curley aborda el tema de las fuentes dice: «a glance at the analogues provided in the notes [...] will show that Physiologus is a syncretic work which draws upon folk legends and pseudoscience common to many Eastern Mediterranean cultures -Greek, Roman, Egyptian, Hebrew, and Indian.» (Physiologus... p.xxi) y se refiere especialmente a la relación con las tradiciones folclóricas egipcias (véase Physiologus... p. XVI, XVIII). Zucker tampoco habla de fuentes específicas y prefiere hablar de la influencia de tradiciones distintas: «Ce livre est au carrefour de cinq traditions: la zoologie grecque, l'ésotérisme égyptien, la mystique juive, l'exégèse alexandrine et la théologie chrétienne du salut.» (Physiologus... p. 25), diferentes estratos que han tenido influencia en la composición y en el desarrollo de los physiologus.

32 Alfonso X el Sabio, General estoria, segunda parte (ed. Belén Almeida, coord. Pedro Sánchez-Prieto 
Esta memoria podría explicar que algunos bestiarios recobren el tema más antiguo del «parir por la boca», pese a que también haya testimonios de la historia del parto de Alcmena que han olvidado por completo el animal en el que Galante había sido metamorfoseada. ${ }^{33}$

Estamos trabajando, pues, en terrenos pantanosos y resbaladizos... En los bestiarios no hay ninguna referencia a Ovidio, a Eliano o a cualquier otra fuente; ninguna de las interpretaciones cotejadas tampoco se refiere a la mitología grecolatina o a tradiciones específicas... esta ausencia impide el establecimiento de lazos concretos y, por lo tanto, obliga a la prudencia. Sin embargo, las similitudes que hemos notado y los testimonios referidos, en particular los pasajes de Eliano (como posible testimonio de la dilución de las materias mitológicas), son elocuentes y parecen indicar la posibilidad de que todos estos textos, physiologus y bestiarios incluso, podrían haberse inspirado en tradiciones míticas que con la llegada del cristianismo se habrían vertido en historias tradicionales disponibles para el uso de distintos autores.

\section{BIBLIOGRAFÍA}

Aarne, Antti y Thompson, Stith, The Types of the Folktale. A Classification and Bibliography, Helsinki, Academia Scientiarum Fennica, 1987.

Alfonso X el SAbio, General estoria, segunda parte (ed. Belén Almeida, coord. Pedro Sánchez-Prieto Borja), Madrid, Fundación José Antonio de Castro, 2009, tomo II.

[ARistote], Histoire des Animaux d'A ristote (trad.fr. par J. Barthélemy-Saint Hilaire), Paris, Hachette, 1883. Disponible en: http://remacle.org/bloodwolf/philosophes/ Aristote/tableanimaux.htm [consultado 13-06-2015].

Beauvais, Vicente de, Vincentius Bellovacensis, Speculum naturale,Venetiis, Hermannus Liechtenstein, 1494.

Berlioz, Jacques y Polo de Beaulieu, Marie Anne (dir.), L'animal exemplaire au Moyen Âge (Ve-XVe siècles), Rennes, Presses Universitaires de Rennes, 1999.

Borja), Madrid, Fundación José Antonio de Castro, 2009, tomo II, pp. 47-48.

33 Este es el caso de la Coronica Troiana em Limguoajem Purtugesa, (ed. Ana María García Martín), Salamanca, Luso-Española de Ediciones, 1998. En este libro se edita y estudia el códice 298 de la Biblioteca Nacional de Portugal, un texto manuscrito del siglo XVI, que deriva de la versión castellana impresa, que a su vez refunde el texto latino de Guido de Columna (Historia destructionis Trojae) y las Sumas de Historia Troyana de Leomarte (García Martín, p. 53): «Des que Juno vio que por emtam não lhe prestauam seus emcamtamentos para se vimgar de sua comboça, soube que nam era verdade o que a domzela chamada Galamte dixera, mas que ho dixera manhosamente porque seus emcamtamentos desfaleçesem. E dizem os autores dos gentios que Juno por aquela manimcorea que contra a domzela tomara que a tornara em coruja. Mas depois se acha em as estorias que foi esta Galamte polo que fez a mais onrrada molher que quantas donas nobres avia em sua terra.» (pp. 179-180). 
Bestiaris (a cura de Saverio Panunzio), Barcelona, Barcino, 1963 (vol. I).

Clark, Willene B. A Medieval Book of Beasts. The Second-Family Bestiary: Commentary, Art, Text and Translation, Woodbridge, The Boydell Press, 2006.

Coronica Troiana em Limguoajem Purtugesa, (ed. Ana María García Martín), Salamanca, Luso-Española de Ediciones, 1998.

Devoto, Daniel «Pisó yerba enconada», Textos y Contextos. Estudios sobre la tradición, Madrid, Gredos, 1974, pp. 11-46.

Duchet Suchaux, Gaston y Pastoureau, Michel Le Bestiaire médiéval. Dictionnaire historique et bibliographique, Paris, Le Léopard d'or, 2002.

Eliano, Claudio, Historia de los animales (trad. de José Vara Donado), Madrid, Akal, 1989.

Fournival, Richard DE, Le Bestiaire d'amour suivi de la réponse de la Dame (ed. C. Hippeau), Ginebra, Slatkine, 1978.

GARCÍA DE LUCAS, CÉSAR, «Iconotropía y literatura medieval», Carlos Alvar (coord.), Estudios de Literatura medieval en la Península Ibérica, San Millán de la Cogolla, Cilengua, 2015, pp. 593-605

Isidoro de Sevilla, Etimologias (ed. bilingüe de Jose Oroz Reta y Manuel A. Marcos Casquero), Madrid, BAC, 1983.

«Le Bestiaire de Gervaise» (ed. Paul Meyer), Romania, 1, 1872, pp. 420-443.

Le Bestiaire de Philippe de Thaün (ed de Emmanuel Walberg), Lund, H.J. Möller - H. Welter, 1900.

Le Bestiaire divin de Guillaume clerc de Normandie, trouvère du XIIIe siècle (ed. C. Hippeau), Ginebra, Slatkine, 1970.

Mermier, Guy R. (trad.), A Medieval Book of Beasts. Pierre de Beauvais' Bestiary, Lewiston / Queenston / Lampeter, The Edwin Mellen Press, 1992.

Ovidio, Metamorfoses (ed. bilingue de Domingos Lucas Dias), Lisboa, Nova Vega, 2008, vol. II.

Pérez, Martín Livro das Confissões (ed. José Barbosa Machado y Fernando Torres Moreira), Matosinhos, Pena Perfeita, 2005.

Physiologos. Le bestiaire des bestiaires (trad. y ed. Arnaud Zucker), Grenoble, Jérôme Millon, 2005.

Physiologus. A Medieval Book of Nature Lore (trad. Michael J. Curley), Chicago \& London, University of Chicago Press, 1979.

Pliny the Elder, The Natural History (ed. John Bostock y H.T.Riley), London, Taylor and Francis, Red Lion Court, Fleet Street, 1855 - disponible en «Perseus Digital Library» (Gregory R. Crane, editor-in-chief, Tufts University) Disponible en http:// data.perseus.org/citations/urn:cts:latinLit:phi0978.phi001.perseuseng1:1.dedication [consultado 01-05-2015]. 
Plutarch, On Isis and Osiris (trad. de Frank Cole Babbitt), ed. Loeb, 1936. Disponible en: http://penelope.uchicago.edu/Thayer/E/Roman/Texts/Plutarch/Moralia/ Isis_and_Osiris*/home.html [consultado 13-06-015].

Solinus, C. Julius, C. Ivlii Solini, Collectanea Rerom Memorabilivm (ed. Th. Mommsen), Berolinum, Weidmann, 1895.

«The Cambrai Bestiary» (trad. ingl. de E. B. Ham), Modern Philology 36/3, 1939, pp. 233-237 (reed. en Guy R. Mermier (trad.), A Medieval Book of Beasts. Pierre de Beauvais' Bestiary, Lewiston/Queenston/Lampeter, The Edwin Mellen Press, 1992, pp. 311-315).

Tratado de Confissom (ed. José Barbosa Machado), Braga, APPACDM, 2003, vol. I.

Vermeille, Alexandre, Physiologus. De l'Orient à l'Occident. Un patchwork multiculturel au service de l'Écriture, Mémoire de latin-Université de Neuchâtel, 2006.

ZUCKER, ARNAUD, «Morale du Physiologos: le symbolisme animal dans le christianisme ancien (IIe-Ve s.)», Rursus, 2, 2007. Disponible en: http://rursus.revues. org/142 DOI: 10.4000/rursus.142 [consultado 01-05-2015]. 